Proceedings of SALT 22: 424-440, 2012

\title{
Avoiding predicate whiplash: social choice theory and linguistic vagueness *
}

\author{
Timothy W. Grinsell \\ University of Chicago
}

\begin{abstract}
Vagueness effects predictably occur in predicates that aggregate judgments along a number of different criteria. With these types of aggregations, small changes along one of the criteria can lead to big changes in the outcome of the aggregation procedure. Vagueness results from avoiding this 'whiplash' effect.
\end{abstract}

Keywords: Semantics, vagueness, social choice, tolerance

\section{Introduction}

This paper attempts an explanation of at least some vagueness effects from the viewpoint of social choice theory. Social choice theory is the branch of economics that has to do with aggregating a number of preferences into one preference, as when many voters select a single candidate. Certain predicates of natural languagelike multidimensional adjectives - involve similar aggregations along a number of criteria (or dimensions). For example, whether a person is described as healthy might depend on their blood pressure, their cholesterol, and other measures that are relevant in context. Results from social choice theory demonstrate that, under some plausible assumptions, the aggregation of judgments along multiple criteria are 'unstable' in the sense that small changes along one criteria can lead to large changes in the outcome of the aggregation procedure. Vagueness effects (in which small changes do not give rise to different judgments, thus engendering the Sorites paradox) result from the avoidance of this instability.

The next section introduces one well-known explanation of vagueness effects from the scale structure of relative and absolute gradable adjectives. Section 3 mounts a challenge to this explanation from multidimensional adjectives like healthy. Section 4 informally introduces the ideas from social choice theory that make tractable the multidimensional challenge. Section 5 applies these ideas to multidimensional additives in order to account for vagueness effects, and section 6 concludes.

* I thank the audience at SALT 22 for their helpful questions and comments. In particular, discussions with Chris Barker, Stephanie Solt, and Galit Sassoon contributed to the improvement of my ideas. Thanks as well to the audience of a workshop at the University of Chicago, in particular Chris Kennedy, Itamar Francez, Peter Klecha, and Julia Thomas.

(C2012 Grinsell 
Avoiding predicate whiplash

\section{Scale structure and vagueness effects}

Kennedy (2007) provides an explanation for vagueness effects based on the 'scale structure' of gradable adjectives. (Roughly speaking, gradable adjectives can appear in comparative constructions while non-gradable adjectives cannot.) First, Kennedy identifies effects typically associated with vagueness. One of these vagueness effects is the existence of borderline cases. These are cases in which we are not sure whether the predicate applies. Adjectives like expensive, for example, will have some things they are clearly true of in a context and some things they are clearly false of. If you're in the market for new bike (perhaps because your old bike was stolen from in front of the University of Chicago), a $\$ 5,000$ bike is clearly expensive and a $\$ 200$ bike, clearly inexpensive. But an $\$ 800$ bike?

A second vagueness effect is the tendency to give rise to the Sorites Paradox, as illustrated in (1).

\section{The Sorites Paradox}

Premise 1: A $\$ 5$ cup of coffee is expensive (for a cup of coffee).

Premise 2: Any cup of coffee that costs 1 cent less than an expensive one is expensive (for a cup of coffee).

Conclusion: Therefore, any free cup of coffee is expensive. (Kennedy 2007:

2)

This argument appears valid, but of course the conclusion is false. The problem seems to be with Premise 2, but it is difficult to explain exactly what that problem is (and why we are so willing to ignore it) (Fara 2000; van Rooij 2011). ${ }^{1}$

With respect to gradable adjectives, Kennedy observed an asymmetry in the display of vagueness effects: while 'relative' gradable adjectives display vagueness effects, 'absolute' gradable adjectives do not. The terms 'relative' and 'absolute' derive from earlier work on the scale structure of gradable adjectives (Kennedy \& McNally 2005; Rotstein \& Winter 2004). This work shows that not all gradable adjectives are alike. Some gradable adjectives are compatible with modifiers like perfectly, while other gradable adjectives are compatible with modifiers like slightly, as shown in (2).

1 Kennedy also identified a third effect: variability in truth conditions. Vague adjectives tend to be context-sensitive, like the adjective expensive in (i).

i. The coffee in Rome is expensive.

(Kennedy 2007: 2)

The sentence in (i) is true if the context is a discussion of coffee prices in Italian cities, but false if the context is a discussion of coffee prices in Chicago and Rome. As Kennedy notes, "This kind of variability is of course not restricted to vague predicates... though all vague predicates appear to display it." (Kennedy 2007: 2). Since this effect is not particular to vague predicates, I omit it in the discussion that follows. 
By adopting a semantics of degree for gradable adjectives, in which gradable adjectives denote functions from individuals to degrees, Kennedy and McNally were able to make sense of this data. On the degree-based account, ${ }^{2}$ the degrees that compose the ranges of gradable adjectives are formalized by a triple $\langle D,<, \delta\rangle$ including the set of degrees $D$, an ordering on this domain $<$, and a dimension $\delta$ that provides the property to be measured (for instance, cost in the case of expensive). Kennedy and McNally explained the data in (2) by arguing that gradable adjectives may differ as to the structure of $D$, and in particular whether $D$ does or does not have maximal or minimal elements. If the $D$ associated with the adjective contains either or both elements, the adjective is 'absolute.' Otherwise, it is 'relative.'

For example, if an adjective's associated set $D$ includes a maximal element, the adjective should be compatible with modifiers that pick out maximal degrees on a scale, like perfectly (see 2c). Similarly, if an adjective's associated set $D$ includes a minimal element, the adjective should be compatible with modifiers that pick out minimal degrees on a scale, like slightly (see 2b). And if the set $D$ includes neither maximal nor minimal elements, the adjective should be compatible with neither modifier (as in 2a), while if the set $D$ contains both maximal and minimal elements, the adjective should be compatible with both modifiers (as in $2 \mathrm{~d}$ ). The following schematizes this 'typology of scale structures':

$\begin{array}{ll}\begin{array}{l}\text { A typology of scale structures } \\ \text { OPEN }\end{array} & \text { (tall, expensive) } \\ \text { LOWER CLOSED } & \text { (bent, dirty) } \\ \text { UPPER CLOSED } & \text { (straight, flat) } \\ \text { (TOTALLY) CLOSED } & \text { (full, open) }\end{array}$

(Kennedy 2007: 33)

Finally, Kennedy observed that the lower closed, upper closed, and totally closed gradable adjectives (that is, the 'absolute' ones) did not display vagueness effects, while the open gradable adjectives (that is, the 'relative' ones) did. In particular, Kennedy demonstrated that absolute adjectives lack borderline cases and do not participate in the Sorites Paradox. ${ }^{3}$ Where two objects differ in length (though both

2 This approach is shared in some form with Cresswell (1977) and von Stechow (1984) among others. It is often contrasted with a delineation approach that treats predicates like expensive as simple predicates with context-dependent extensions (Kamp 1975; Klein 1980).

3 Absolute adjectives do display 'imprecision,' but a discussion of the imprecision vs. vagueness debate would take us too far afield. 
Avoiding predicate whiplash

may be short or long when judged individually), for instance, experiment subjects readily accepted a definite description with a relative adjective to pick out the longer one, as in (4).

(4) Pass me the long one.

In contrast, subjects rejected this use with absolute adjectives like full. If neither of two cups was filled to the top, even though the cups contained differing amounts of liquid, subjects rejected sentences like (5) (Syrett, Kennedy \& Lidz 2010).

(5) Pass me the full one.

These results indicate that absolute adjectives like full have endpoint-oriented interpretations - and, in turn, that they have no true borderline cases because there is a sharp cutoff between the endpoint degree and the non-endpoint degrees. In other words, it is easy to tell on what side of the border a particular object falls. Additionally, absolute adjectives resist the Sorites Paradox in a way that relative adjectives do not. In (6), for example, the second premise is not as readily acceptable as it is in (1).

(6) Premise 1: A rod that has 10 degrees of bend is bent.

Premise 2: A rod that is 1 degree less bent than a bent rod is bent.

Conclusion: A rod that has 0 degrees of bend is bent.

These observations led Kennedy to propose an explanation of vagueness as a function of 'standing out.' What it means to 'stand out' is to be on the upper end of a natural transition, a transition from a minimal degree to a non-minimal degree (as in the case of bent) or from a non-maximal degree to a maximal degree (as in the case of full) (Kennedy 2007: 32). Kennedy represented this relation in the gradable adjective's 'standard of comparison': an entity is tall, for instance, if its degree of height exceeds the degree necessary to stand out in context.

$$
\begin{aligned}
& \llbracket \text { tall } \rrbracket \\
& \lambda x . \operatorname{tall}(\mathrm{x}) \geq \mathbf{s}(\text { tall })
\end{aligned}
$$

a. The function $\mathbf{s}$ chooses a standard of comparison in such a way as to ensure that the objects that tall is true of 'stand out' in the context of utterance, relative to the kind of measurement that the adjective encodes.

b. ' $\mathrm{x}$ is tall' is true iff $\mathrm{x}$ stands out in context relative to the comparison class

If Kennedy's definition of standing out is right, then absolute adjectives - those with endpoints to their scale structure-always have a potential degree that stands out. Relative adjectives, on the other hand, do not. Kennedy then adopted a principle of 'Interpretive Economy' that says this: if a stand-out degree is part of the scale 
structure, that degree is usually chosen by the degree function. ${ }^{4}$ This correctly derives Kennedy's observation. Absolute adjectives do not display vagueness effects because the predicated-of entity always stands out. The meanings of a relative adjective like tall as in (7) and an absolute adjective like straight then differ in the following way:

$$
\begin{aligned}
& \llbracket \text { straight } \rrbracket \\
& \lambda x \text {.straight }(\mathrm{x}) \geq \mathbf{s}(\text { straight })
\end{aligned}
$$

a. The function s chooses a standard of comparison in such a way as to ensure that the objects that straight is true of 'stand out' in context. In the case of an absolute adjective like straight, Interpretive Economy ensures that this standard is always the endpoint of the scale.

b. ' $\mathrm{x}$ is straight' is true iff $\mathrm{x}$ stands out in context relative to the comparison class.

On Kennedy's account, therefore, the two adjectives have similar denotations, but the 'stand out' relation is computed differently.

Kennedy's analysis also answered to Fara's (2000) criteria for a successful analysis of vagueness. First, Kennedy explained why the Sorites premise is false: there is a precise cut-off point of the 'stand-out' relation in context, though we don't always know where it is (see also Williamson 1994). Second, we accept the Sorites premise as true because rejecting it involves invoking a context in which objects that differ minimally 'stand out' with respect to one another, and this is odd.

\section{The challenge from multidimensional adjectives}

Since Kennedy's analysis of vagueness depends crucially on scale structure, we might ask whether it is possible to pry apart scale structure and vagueness effects. As this section shows, there are some absolute adjectives that still display vagueness effects, contrary to Kennedy's predictions. These adjectives are 'multidimensional' adjectives.

Multidimensional adjectives are associated with multiple criteria (or dimensions in Kennedy's sense) (Kamp 1975; Klein 1980; Sassoon To appear). For example, similar, identical, typical, normal, good, happy, human, and healthy are all multidimensional adjectives. Whether Clarence is healthy intuitively depends on a number of factors simultaneously, like blood pressure, heart function, cholesterol levels, and still other measures. Context normally supplies the relevant dimensions.

4 The actual formulation of Interpretive Economy is less ad hoc: "Maximize the contribution of the conventional meanings of the elements of a sentence to the computation of its truth conditions" (Kennedy 2007: 36). 
Avoiding predicate whiplash

Sassoon (To appear) provides a number of ways to identify multidimensional adjectives. For example, it is possible to specify a dimension overtly with prepositional phrases like with respect to or in, as in (9). Such specifications are not available for unidimensional adjectives.

a. John is healthy with respect to his blood pressure.

b. * The wedding is long \{with respect to, in $\}$ temporal duration (but not with respect to space).

It is also possible to quantify over multiple dimensions, as in (10).

(10) a. Elena is healthy in \{every respect, some respects, most respects .

b. * The table is long in $\{$ all, most, three, some $\}$ respects.

And it is possible to except dimensions from consideration, as in (11).

(11) a. Ruth is healthy except with respect to her cholesterol.

b. * The table is long except with respect to temporal duration.

These tests suggest that an adjective like healthy is sensitive to multiple dimensions, whether as overt semantic arguments (as Sassoon suggests) or otherwise.

Moreover, some multidimensional adjectives pattern with absolute adjectives, as in (12a). An adjective like healthy is compatible with the modifier perfectly, which picks out the maximal element in a set of degrees. It is not (or at least not as) compatible with the modifier slightly, which requires a minimal element in a set of degrees. This is the same pattern that upper closed adjectives like straight display. The multidimensional adjective abnormal follows the same patterns as lower closed adjectives like bent (see 12b). Still other multidimensional adjectives like familiar follow the totally closed patterns, as in (12c).

(12) a. Stephen is \{perfectly, almost, ??slightly\} healthy.

b. Tony is $\{$ ??perfectly, slightly\} abnormal.

c. Ruth is \{perfectly, slightly\} familiar with these routines.

These multidimensional adjectives also behave like absolute adjectives in the definite description test. Imagine two sickly looking plants, one much more sickly looking than the other. My intuition is to reject (13).

(13) ?? Pass me the healthy one.

Syrett et al. (2010)

But if these tests suggest an endpoint-oriented interpretation, as with absolute adjectives, they do not signal a lack of vagueness effects. In particular, multidimensional adjectives like healthy behave in a way that is inconsistent with Interpretive Economy. For instance, a totally closed multidimensional adjective like familiar may have a non-endpoint-oriented interpretation, as the parenthetical followup to (14) demonstrates. 
(14) For a new Justice, Elena is familiar with the Court's routines. (In fact, she's completely familiar with them.) McNally (2011)

More importantly, endpoint-oriented multidimensional adjectives display vagueness effects like borderline cases (as in 15) and Sorites premises (as in 16).

(15) A person with low blood pressure, low cholesterol, and a low heart rate is healthy. A person with high blood pressure, high cholesterol, and a high heart rate is not healthy. Is a person with low blood pressure, low cholesterol, and a high heart rate healthy? A person with mid-values for all the measures? ${ }^{5}$

(16) A person whose systolic blood pressure is one unit higher than a healthy person's is healthy.

Thus, the absolute/relative adjective distinction does not account for the vagueness effects exhibited by some multidimensional adjectives. Vagueness in multidimensional adjectives demands a different explanation.

\section{An introduction to social choice theory}

Vagueness in multidimensional adjectives is a product of the aggregation of rankings of members of the comparison class along those multiple dimensions. In order to explain this explanation, though, it is necessary to first introduce some results from social choice theory. These results illuminate the difficulties inherent in making judgments along a number of criteria and then distilling those judgments into one decision. In such aggregations, the final decision respects transitivity or continuity, but not both. Multidimensional adjectives respect transitivity at the price of discontinuity-the source of their vagueness effects.

\subsection{Intransitivity}

Suppose that a local community's legislature is voting on how much to fine litterers. ${ }^{6}$ The legislature is composed of 3 equal voting blocs - the liberals, the conservatives, and the moderates. The proposals available are a $\$ 1,000$ fine, a $\$ 100$ fine, and no fine (a $\$ 0$ fine). The liberals prefer a stiff fine, but for them any fine is better than no fine. The conservatives prefer no fine, but if a fine is imposed, it should be punitive. And the moderates prefer a moderate fine, but they would never support an exorbitant fine. The following table shows the voters' preferences:

5 Sassoon claims that healthy means something like 'healthy in all respects,' so an unhealthy measure on one dimension disqualifies the entity from being healthy. My own intuitions are not robust here, though even if Sassoon is correct, there are still borderline cases of healthy (e.g. the person with mid-values for all the measures).

6 This example is a modified version of that presented in Katz 2011. 
Avoiding predicate whiplash

(17)

\begin{tabular}{|l|l|l|l|}
\hline & liberals & conservatives & moderates \\
\hline first best & $\$ 1,000$ & $\$ 0$ & $\$ 100$ \\
\hline second best & $\$ 100$ & $\$ 1,000$ & $\$ 0$ \\
\hline third best & $\$ 0$ & $\$ 100$ & $\$ 1,000$ \\
\hline
\end{tabular}

What proposal wins? Evaluating the options pairwise, the liberals and the conservatives will team up to vote for the $\$ 1,000$ proposal over the $\$ 100$ proposal,

\begin{tabular}{|l|l|l|l|l|}
\hline & liberals & conservatives & moderates & winner \\
\hline$\$ 100$ or $\$ 1,000 ?$ & $\$ 1,000$ & $\$ 1,000$ & $\$ 100$ & $\$ 1,000$ \\
\hline
\end{tabular}

the conservatives and the moderates will team up to vote for the $\$ 0$ proposal over the $\$ 1,000$ proposal,

\begin{tabular}{|l|l|l|l|l|}
\hline & liberals & conservatives & moderates & winner \\
\hline$\$ 1,000$ or $\$ 0 ?$ & $\$ 1,000$ & $\$ 0$ & $\$ 0$ & $\$ 0$ \\
\hline
\end{tabular}

and the moderates and the liberals will team up to vote for the $\$ 100$ proposal over the $\$ 0$ proposal.

\begin{tabular}{|l|l|l|l|l|}
\hline & liberals & conservatives & moderates & winner \\
\hline$\$ 0$ or $\$ 100 ?$ & $\$ 100$ & $\$ 0$ & $\$ 100$ & $\$ 100$ \\
\hline
\end{tabular}

So the legislature has the following preferences in the following order: $\$ 100$ fine $>$ $\$ 0$ fine $>\$ 1,000$ fine $>\$ 100$ fine. These preferences 'cycle'. 7

Cycling is a failure of transitivity-the principle that if $a R b$ and $b R c$, then $a R c$ for any relation $R$. And it turns out that any method of aggregating ordinal rankings that respects certain weak assumptions will either cycle or converge to dictatorship, in which one voter's ranking always wins. This result is Arrow's Theorem. Endriss (2011) explains:

Arrow argued that any acceptable method of aggregation should satisfy at least the following two axioms:

i. If every individual ranks $x$ above $y$, then so should society.

ii. It should be possible to determine the relative social ranking of $x$ and $y$ by considering only the relative ranking of $x$ and $y$ supplied by each of the individuals. ${ }^{8}$

7 This is the Condorcet paradox, of which Arrow's Theorem (below) is a generalization.

8 ED: This assumption is the subject of an extensive literature, but I will put aside these concerns. See (Berg 1996; Estlund 1994; Dietrich \& List 2004; Dietrich 2008). 
... Arrow then proved a truly astonishing result: If there are at least three alternatives, then the only kind of mechanism that will respect both of our axioms and that will return a collective preference that is a linear order for any combination of individual preferences is a dictatorship, i.e., a function that simply copies the preferences of a fixed individual and returns it as the collective preference! In other words, satisfying both axioms and the requirement of being nondictatorial is impossible.

Endriss's 'linear order' is an order that respects transitivity. Arrow demonstrated that transitivity and the principle of non-dictatorship are in tension. The price of retaining both principles in an aggregation method is admitting discontinuity.

\subsection{Discontinuity}

An aggregation function (like the majority vote our litter-conscious legislature used) can retain transitivity, unanimity (as in i), and nondictatoriality, but only at the price of discontinuity. Loosely, 'continuity' requires that a small change in the domain of a function does not yield a big change in the range of the function (Gaertner 2006). Aggregation functions do not have this property. For example, imagine that five people are evaluating the options $v, x, y$, and $z(21)$.

\begin{tabular}{|lllll|}
\hline John & Ruth & Sonia & Clarence & Stephen \\
$x$ & $y$ & $z$ & $x$ & $z$ \\
$y$ & $v$ & $v$ & $v$ & $x$ \\
$z$ & $x$ & $y$ & $z$ & $v$ \\
$v$ & $z$ & $x$ & $y$ & $y$ \\
\hline
\end{tabular}

In a situation like (21), $x$ is a decisive winner, meaning that $x$ is the option that, when compared with every other option, is preferred by more people. ${ }^{9}$ But if we switch Ruth's rankings of $x$ and $z$, then $z$ becomes a decisive winner. That is, a small change among the least preferred options in Ruth's ordering yields quite a big change in the outcome of the aggregation procedure (Gaertner 2006: 168).

Chichilnisky formalized this result.

\section{Chichilnisky's Theorem}

A continuous aggregation rule cannot simultaneously satisfy transitivity, nondictoriality, and unanimity.

Chichilnisky (1982)

9 This is known as the Condorcet winner. We can actually say more about $x$ : it is also the winner according to the Borda method, in which options are assigned points based on their position in a person's ranking (5 points for first place, etc.). The option with the most overall points in a Borda count is the winner. 
Chichilnisky's proof is complicated, but Baigent (2011) provides a helpful illustration: a beach party! Assume two people, Sam and Elena, want to have a party on the shore of a perfectly round lake. Sam selects location $s^{\prime}$, and Elena selects location $s^{\prime \prime}$. The function $f$ aggregates these preferences, picking the best spot based on Sam and Elena's choices.

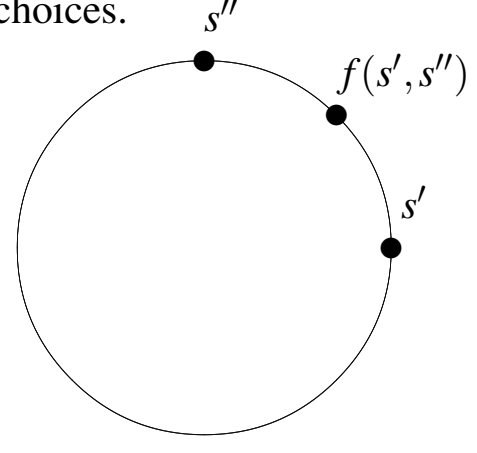

As Elena's choice moves towards 9 o'clock, $f$ will move in that direction as well. But as soon as Elena moves past the 9 o'clock mark, $f$ moves to the other side of the lake.

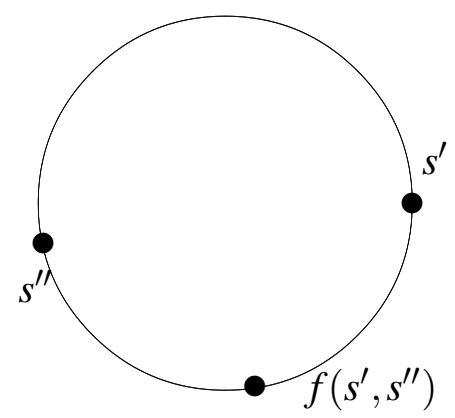

The function $f$ 's big jump, based on a small change in the position of $s^{\prime \prime}$, is an example of discontinuity. What Chichilnisky showed was that as long as an aggregation function like $f$ respects unanimity and non-dictatorship, $f$ will be discontinuous. ${ }^{10}$

According to Arrow and Chichilnisky's results, if an aggregation function respects transitivity, then small changes in the input to this function will result in large changes to the output. This is where vagueness phenomena come from in multidimensional adjectives. ${ }^{11}$

10 Chichilnisky actually proved this result for a slightly stronger condition than non-dictatorship, but this detail doesn't effect the analysis to follow.

11 For other discussions on the relation between Sorites-type vagueness and social choice theory, see Katz 2011; Ackerman 1994. For a discussion of vagueness that uses Arrow's results in a different way, see van Rooij 2011. 


\section{Explaining vagueness in multidimensional adjectives}

This section applies social theory to an analysis of vagueness in multidimensional adjectives. It proceeds in three parts: first, extending the results applicable to collective decision making (Arrow, Chichilnisky) to individual decision making; second, demonstrating that the use of an adjective like healthy is just such an individual 'decision'; and third, explaining how applying the social choice results to these individual 'decisions' generates vagueness.

We can draw an analogy between voting and multicriterial decision making. (Arrow \& Raynaud 1986; Hurley 1992; Sen 1977; Feldman \& Serrano 2008). For example, if we want to model how an individual chooses a car, we can replace 'voters' with 'criteria' and 'proposals' with 'alternatives.' The individual deciding between a Ford, a Chevy, and a Chrysler ranks them according to price, comfort, and style.

\begin{tabular}{|l|l|l|l|}
\hline & price & comfort & style \\
\hline first best & Ford & Chrysler & Chevy \\
\hline second best & Chevy & Ford & Chrysler \\
\hline third best & Chrysler & Chevy & Ford \\
\hline
\end{tabular}

Arrow and Chichilnisky's results will hold for this decision procedure just as well as they hold for collective decision procedures. Indeed, they will hold for any aggregation function that respects the axioms identified by Endriss (that is, $i$ and ii). This includes aggregations of ordinal rankings along multiple criteria-what I expect an adjective like healthy does.

As represented in (26), for example, the adjective healthy is an aggregation function that ranks members of the comparison class along the several relevant dimensions and spits out a single, aggregated ranking.

\begin{tabular}{|l|l|l|l|}
\hline healthy & bp & cholesterol & heart rate \\
\hline first best & John & John & Ruth \\
\hline second best & Ruth & Stephen & Stephen \\
\hline third best & Stephen & Ruth & John \\
\hline
\end{tabular}

The healthy entities are those toward the top of this final ranking, and the unhealthy entities are those toward the bottom. ${ }^{12}$

If this is right, then multidimensional adjectives must not depend on some sort of impressionistic similarity measure for their use. And they don't seem to: Sassoon

12 Exactly where one draws the line between the healthy and the unhealthy entities on the final ranking is not important. Even if the semantics specified that the line must be drawn between the top-ranked entity and the rest of the entities, the ranking itself would still be subject to Arrowian difficulties. These are the difficulties that give rise to vagueness effects. 
Avoiding predicate whiplash

(To appear) provides evidence that dimensions in multidimensional adjectives are aggregated through 'logical, Boolean' operations. Moreover, as Égré \& Klinedinst (2011) rightly worry, multidimensional adjectives may possibly give rise to "intransitivities of a kind familiar in social choice theory ( $a$ may be judged more clever than $b$ along one dimension, $b$ more clever than $c$ along another, and $c$ more clever than $a$ along a third)."

Accepting Égré and Klinedinst's invitation to social choice theory, it appears that multidimensional adjectives obey the Arrowian axioms, at least intuitively. For instance, if every criterion that factors into a use of healthy ranks Elena above other people, then a felicitous use of healthy should so reflect. The sentence in (27) provides some support for this.

$$
\text { ?? Elena is healthy in every respect, but she is not healthy. }{ }^{13}
$$

In addition, it should be possible to determine the relative health of Nino and Ruth by considering only the relative ranking of Nino and Ruth supplied by the health criteria.

(28) A: Nino is healthier than Ruth with respect to blood pressure and cholesterol.

B: Stephen is less healthy than Ruth with respect to blood pressure.

A: ??Then Nino is less healthy than Ruth.

If multidimensional adjectives respect the Arrowian axioms, then multidimensional adjectives are subject to Arrow and Chichilnisky's results.

Given Chichilnisky's result, if the final aggregated ranking produced by the predicate healthy is transitive, then it is necessarily discontinuous. Multidimensional adjectives do appear to respect transitivity, as in (29).

(29) Clarence is healthier than Sonia and Sonia is healthier than Tony \{?and Tony is healthier than Clarence\}.

Thus, multidimensional adjectives must be discontinuous. This means a small change along one of the criteria (blood pressure, say) could dramatically affect which entities appear at the top of the healthy ranking and therefore which entities healthy is true of.

Speakers avoid making fine distinctions with multidimensional predicates in order to avoid discontinuity. A fine distinction with a predicate like healthy would involve a distinction along one of its dimensions, and a speaker could not be sure that this small difference wouldn't alter the entire ranking (what I will call 'predicate whiplash'). ${ }^{14}$ That is, a seemingly insignificant distinction could have a significant

13 This sentence is acceptable in the situation where we can't figure out the source of Elena's illness, but then we're simply missing a criteria in our aggregation.

14 This sort of explanation may also account for the 'principal of tolerance' associated with vague predicates. 
effect, so speakers avoid making seemingly insignificant distinctions. This results in vagueness effects. In (30), for instance, five criteria (instead of five people, as in 21) rank members of the comparison class $v, x, y$, and $z$.

\begin{tabular}{|lllll|}
\hline C1 & C2 & C3 & C4 & C5 \\
$x$ & $y$ & $z$ & $x$ & $z$ \\
$y$ & $v$ & $v$ & $v$ & $x$ \\
$z$ & $x$ & $y$ & $z$ & $v$ \\
$v$ & $z$ & $x$ & $y$ & $y$ \\
\hline
\end{tabular}

And as in (21), a small difference among low ranked options according to criteria 2 (C2) can change the output of the aggregated ranking. That is, this small difference results in predicate whiplash.

Speakers avoid predicate whiplash in order to maintain a predictably stable common ground (Stalnaker 1978). Whatever view of the common ground one accepts, its usefulness is diminished when seemingly insignificant changes to it can alter the truth of propositions in it. ${ }^{15}$ So speakers and hearers avoid making distinctions that undermine robust reliance on the truth of accepted propositions-the common ground.

Now we are in a position to account for Fara's (2000) criteria of a successful analysis of vagueness. The Sorites premise is false because, by Chichilnisky's discontinuity result, at some point a small change in an entity along one dimension will change an entity's position near the top of the aggregated ranking to a position nearer the bottom of the aggregated ranking. As Williamson (1994) argues, there is a cut-off point, for example, between the healthy and the unhealthy entities. However, we accept the Sorites premise as true because we avoid predicate whiplash - the cost of error in making the wrong fine distinctions with multidimensional predicates is too high.

\section{Conclusion}

It is tempting to try to extend this account to the meaning of tall and other vague, unidimensional adjectives. On the one hand, Kennedy's tests for the absolute/relative

15 Seen in this light, the tendency to avoid predicate whiplash may be a corollary of our tendency to avoid irrelevant alternatives - one of Arrow's axioms (see ii). The old joke on irrelevant alternatives goes like this:

i. Waiter: We have chicken and fish.

Patron: I'll have the fish.

Waiter: Sorry, I forgot that we also have steak.

Patron: Well, then I'll have the chicken! 
distinction demonstrate that lexically associated scales play a role in vagueness phenomena for these adjectives. On the other hand, the 'stand out' relation does precisely what I've argued a multidimensional adjective does: ignores small differences along a dimension.

One possible extension is the following: multiple methods are simultaneously at work in calculating the stand-out relation for relative gradable adjectives like tall. Instead of multiple dimensions, relative adjectives like tall aggregate rankings of the comparison class according to different ways of setting the standard of comparison, like pairwise comparisons, determining the average, etc. Schmidt, Goodman, Barner \& Tenenbaum (2009) provides evidence that standards are computed based on an unknown nonparametric function, which is consistent with this hypothesis. Moreover, the interpretation of relative adjectives is susceptible to framing effects in which the hearer is biased toward one method of calculating the standard over others (Yalcin 2012). As long as at least two methods work together to determine the standard of comparison, Arrow and Chichilnisky's results anticipate the same aggregation problems for relative unidimensional predicates as for multidimensional predicates.

The social choice approach may also explain why absolute unidimensional adjectives do not display vagueness effects. These adjectives do not involve multiple methods of computing the stand-out relation. The lexically associated scale provides a ready method for computing the stand-out relation because it includes at least one endpoint, and the endpoint stands out. But since absolute adjectives therefore do not employ more than one 'criteria' in their semantics, they are not subject to aggregation problems. Thus, they should not display vagueness effects.

The difference between absolute and relative adjectives is then a function of how many 'criteria' figure in the computation of the stand-out relation. Scale structure is relevant because it eliminates the need (in absolute adjectives) to compute this relation in multiple ways.

More could (and should) be said about almost all of this. For example, Arrow's Theorem only applies where alternatives are ordered according to ordinal—and not cardinal-rankings. Therefore, this account is not directly applicable where some criteria weigh more than others in determining the ultimate ranking. In addition, if this approach is right, there should be more evidence that multidimensional adjectives work by aggregating judgments among criteria, just like there should be more evidence that relative adjectives work by aggregating rankings along various standards of comparison. And this paper only skims the surface of the relation between vagueness and the common ground when viewed from a social choice perspective.

For all these drawbacks (and others unmentioned or unnoticed), a social choice approach to vagueness is probably on the right track. Both the property of vagueness 
and social choice functions are allergic to small, seemingly irrelevant changes, and this allergy motivates most analyses of vagueness (see, e.g. van Rooij 2011). Moreover, this account makes strong predictions that are easy to test: if it is right, there should be no non-vague multidimensional adjectives that appear to weigh criteria evenly. The social choice approach also presents a possible avenue toward a theory of vagueness applicable to multiple lexical categories. After all, vagueness is not only a property of gradable adjectives. Perhaps a noun like chair can be vague in the proper context because its usage invokes a number of criteria for chairness, not all of which agree.

These projects must be left to future work. For the present, one conclusion is clear: avoid predicate whiplash; it is bad for your health.

\section{References}

Ackerman, Felicia. 1994. Roots and consequences of vagueness. Philosophical Perspectives 8. 129-136.

Arrow, Kenneth \& Herve Raynaud. 1986. Social choice and multicriterion decisionmaking. MIT Press Books 1.

Baigent, Nicholas. 2011. Topological theories of social choice. Handbook of Social Choice and Welfare 2. 301-334.

Berg, Sven. 1996. Condorcet's jury theorem and the reliability of majority voting. Group Decision and Negotiation 5(3). 229-238.

Chichilnisky, Graciela. 1982. Social aggregation rules and continuity. The Quarterly Journal of Economics 97(2). 337-352.

Cresswell, M.J. 1977. The semantics of degree. In B. Partee (ed.), Montague Grammar, 261-292. Academic Press.

Dietrich, Franz. 2008. The premises of Condorcet's jury theorem are not simultaneously justified. Episteme 5(01). 56-73.

Dietrich, Franz \& Christian List. 2004. A model of jury decisions where all jurors have the same evidence. Synthese 142(2). 175-202.

Égré, Paul \& Nathan Klinedinst. 2011. Vagueness and Language Use. Palgrave Macmillan.

Endriss, Ulle. 2011. Logic and social choice theory. In A. Gupta \& J. van Bentham (eds.), Logic and Philosophy Today, U. Amsterdam.

Estlund, David. 1994. Opinion leaders, independence, and Condorcet's jury theorem. Theory and Decision 36(2). 131-162.

Fara, Delia Graff. 2000. Shifting sands: An interest-relative theory of vagueness. Philosophical Topics 28(1). 45-82. Originally published under the name 'Delia Graff'.

Feldman, Allan \& Robert Serrano. 2008. Arrow's impossibility theorem: Two simple 
Avoiding predicate whiplash

single-profile versions. Brown University Department of Economics Working Paper No. 2006-11.

Gaertner, Wulf. 2006. A Primer in Social Choice Theory. Oxford.

Hurley, S.L. 1992. Natural reasons: Personality and polity. Oxford University Press, USA.

Kamp, Hans. 1975. Two theories about adjectives. Formal Semantics of Natural Language 123-155.

Katz, Leo. 2011. Why the Law is so Perverse. University Of Chicago Press.

Kennedy, Christopher. 2007. Vagueness and grammar: The semantics of relative and absolute gradable adjectives. Linguistics and Philosophy 30(1). 1-45.

Kennedy, Christopher \& Louise McNally. 2005. Scale structure, degree modification, and the semantics of gradable predicates. Language 345-381.

Klein, Ewan. 1980. A semantics for positive and comparative adjectives. Linguistics and philosophy 4(1). 1-45.

McNally, Louise. 2011. The relative role of property type and scale structure in explaining the behavior of gradable adjectives. Vagueness in Communication 151-168.

van Rooij, Robert. 2011. Vagueness and linguistics. Vagueness: A Guide 123-170.

Rotstein, Carmen \& Yoad Winter. 2004. Total adjectives vs. partial adjectives: Scale structure and higher-order modifiers. Natural Language Semantics 12(3). 259-288.

Sassoon, Galit Weidman. To appear. A typology of multidimensional adjectives. Journal of Semantics .

Schmidt, L.A., N.D. Goodman, D. Barner \& J.B. Tenenbaum. 2009. How tall is tall? Compositionality, statistics, and gradable adjectives. In Proceedings of the 31st Annual Conference of the Cognitive Science Society, .

Sen, Amartya. 1977. On weights and measures: informational constraints in social welfare analysis. Econometrica: Journal of the Econometric Society 1539-1572.

Stalnaker, Robert. 1978. Assertion. Syntax and Semantics: Pragmatics 9.

von Stechow, Arnim. 1984. Comparing semantic theories of comparison. Journal of Semantics 3(1-2). 1-77.

Syrett, Kristen, Christopher Kennedy \& Jeffrey Lidz. 2010. Meaning and context in children's understanding of gradable adjectives. Journal of semantics 27(1). $1-35$.

Williamson, Timothy. 1994. Vagueness. Routledge: New York.

Yalcin, Seth. 2012. Alternatives in the analysis of epistemic and deontic modals. Presentation at UChicago Semantics and Philosophy of Language Workshop. 
Grinsell

Timothy W. Grinsell

University of Chicago

1010 E. 59th St.

Chicago, IL 60637

twg@uchicago.edu 